\title{
Status of LIGO detectors and results from recent astrophysical searches
}

\author{
Vuk Mandic* for the LIGO Scientific Collaboration \\ Caltech, USA \\ E-mail: mandic_v@ligo.caltech.edu
}

\begin{abstract}
The Laser Interferometer Gravitational-wave Observatory (LIGO) has built three multi-km scale interferometers, designed to search for gravitational waves. The sensitivity of the LIGO interferometers has been rapidly increasing over the recent years, allowing a number of searches for sources of gravitational waves to be conducted. We discuss the current status of the LIGO interferometers and the results from the recent search for stochastic background of gravitational waves. For the frequency independent gravitational wave spectrum, we report a new Bayesian $90 \%$ UL on the spectral amplitude of $8.4 \times 10^{-4}$ in the frequency range $69-156 \mathrm{~Hz}$.
\end{abstract}

International Europhysics Conference on High Energy Physics

July 21st - 27th 2005

Lisboa, Portugal

${ }^{*}$ Speaker. 
LIGO has built three multi-km interferometers: $4 \mathrm{~km}(\mathrm{H} 1)$ and $2 \mathrm{~km}(\mathrm{H} 2)$ at Hanford, WA, and $4 \mathrm{~km}$ (L1) in Livingston Parish, LA. The interferometers are sensitive to quadrupolar oscillations in the space-time metric due to a passing gravitational wave $(\mathrm{GW})$, and can therefore directly measure the strain amplitude of gravitational waves. The detector configuration and performance during the first LIGO science run (S1) is described in [1]. Each interferometer contains a Fabry-Perot cavity in each of the arms, effectively increasing the arm lengths (and improving the strain sensitivity) by $\sim 100$ times. Furthermore, light reflecting back from the interferometer is recycled again (using the power-recycling mirror), effectively increasing the input laser power by a factor of $\sim 40$. All the mirrors are freely suspended, and their length and angular positions are controlled by a collection of feedback servos. The strain sensitivity of these interferometers is limited by several fundamental noise sources: seismic noise (below $\sim 40 \mathrm{~Hz}$ ), thermal noise of the mirrors and suspension wires $(40-150 \mathrm{~Hz})$, and shot noise $(>150 \mathrm{~Hz})$. In practice, a number of other, technical noise sources have appeared, and much of the commissioning effort has focused on identifying and removing these noise sources. In particular, tuning the servos for the angular and auxiliary length degrees of freedom, increasing the laser power, compensating for thermal lensing of the heated mirrors, controlling the laser intensity and frequency noise, reducing the phase noise of the local oscillators, and implementing active seismic suppression system (L1 only) have allowed a 3-4 orders of magnitude increase in sensitivity over the last four years, as shown in Figure 1 (left).
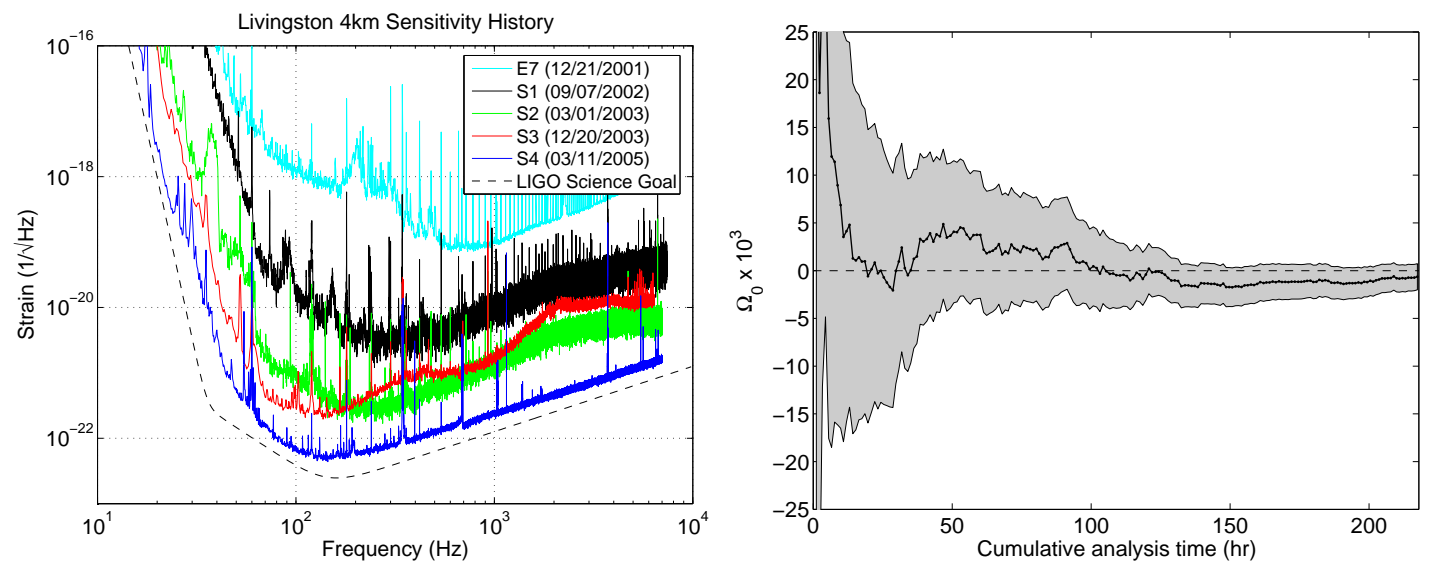

Figure 1: Left: Strain power spectra for L1 in the engineering run E7, and in the fi rst four science runs (S1 - S4). Right: estimate of $\Omega_{0}$ as a function of the amount of the $S 3$ data analyzed. The shaded region shows the $\pm 2 \sigma_{\Omega_{0}}$ band.

The GW stochastic background is usually described in terms of the logarithmic spectrum:

$$
\Omega_{\mathrm{GW}}(f)=\frac{1}{\rho_{c}} \frac{d \rho_{\mathrm{GW}}}{d \ln f}
$$

where $\rho_{\mathrm{GW}}$ is the energy density of gravitational waves, $\rho_{c}$ is the critical density of the Universe, and $f$ is frequency. Many possible sources of such a background have been proposed, both cosmological and astrophysical (see [2,3] for reviews). We define the following cross-correlation estimator [4]:

$$
Y=\int_{-\infty}^{+\infty} Y(f) d f=\int_{-\infty}^{+\infty} d f \int_{-\infty}^{+\infty} d f^{\prime} \delta_{T}\left(f-f^{\prime}\right) \tilde{s}_{1}(f)^{*} \tilde{s}_{2}\left(f^{\prime}\right) \tilde{Q}\left(f^{\prime}\right),
$$


where $\delta_{T}$ is a finite-time approximation to the Delta function, $\tilde{s}_{1}$ and $\tilde{s}_{2}$ are the Fourier transforms of the strain time-series of two interferometers, and $\tilde{Q}$ is the optimal filter. Assuming that the detector noise is Gaussian, stationary, uncorrelated between the two interferometers, and uncorrelated with and much larger than the GW signal, the variance of the estimator $Y$ is given by:

$$
\sigma_{Y}^{2} \approx \frac{T}{2} \int_{0}^{+\infty} d f P_{1}(f) P_{2}(f)|\tilde{Q}(f)|^{2}, \text { where } \tilde{Q}(f)=N \frac{\gamma(f) S_{G W}(f)}{P_{1}(f) P_{2}(f)} .
$$

Here, $P_{i}(f)$ are the power spectral densities (PSDs) of the two interferometers, and $T$ is the measurement time. The form of the optimal filter $\tilde{Q}(f)$ is obtained by optimizing the signal-to-noise [4]. $\gamma(f)$ is the overlap reduction function (due to the locations and orientations of the two interferometers), and $S_{G W}(f)=\left(3 H_{0}^{2} / 10 \pi^{2}\right) f^{-3} \Omega_{t}(f)$ is the template strain power spectrum to be searched. We assume a power law for $\Omega_{t}(f)=\Omega_{\alpha}(f / 100 \mathrm{~Hz})^{\alpha}$, and we choose the normalization constant $N$ such that $\langle Y\rangle=\Omega_{\alpha} T$.

We apply this algorithm to the H1-L1 pair data from the LIGO science run S3, which took place between 31 Oct 2003 and 9 Jan 2004. The sensitivity of the interferometers was significantly improved as compared to the previous LIGO science runs [5] (see Fig. 1 (left)). We divide the data into 60 -sec intervals. Each interval is down-sampled to $1024 \mathrm{~Hz}$, high-pass filtered $(40 \mathrm{~Hz}$ cut-off), and Hann-windowed to avoid spectral leakage from strong lines that may be present in the data. Since Hann-windowing effectively reduces the interval length by a factor of 2, the intervals are 50\% overlapped to recover the loss in signal-to-noise. We calculate the strain power spectral densities (PSDs), using Welch's modified periodogram method on 58 periodograms formed from 4 sec-long, 50\% overlapping data windows. The PSDs for a given interval are calculated using the two neighboring intervals. This avoids a bias in the cross-correlation that would otherwise exist, due to non-zero covariance between the cross-spectrum and the corresponding power spectra. It also allows identifying and rejecting intervals with noise transients, by comparing $\sigma_{Y}^{2}$ calculated in this way with $\sigma_{Y}^{2}$ calculated using the interval itself - we reject about $20 \%$ of the data by requiring that the two estimates of $\sigma_{Y}^{2}$ agree within 20\%, leaving 218 hours of integrated exposure time.

For each interval, the data is calibrated in the frequency domain using the interferometer response functions. These functions are calculated once per $60 \mathrm{sec}$ by measuring the interferometer response to a sinusoidal calibration force. The data is then rebinned into $1 / 4 \mathrm{~Hz}$ resolution, to match the optimal filter binning. Frequencies with known instrumental correlations are excluded from the analysis. This includes the $60 \mathrm{~Hz}$ harmonics, simulated pulsar lines, and $16 \mathrm{~Hz}$ harmonics (arising from slight but periodic $(16 \mathrm{~Hz})$ data corruption at each site - a problem corrected after S3), amounting to $2 \%$ loss in bandwidth.

For each interval we complete the calculations of Eqs. 2 and 3. We then calculate a weighed average of $Y(f)$ over all intervals, with $1 / \sigma_{Y}^{2}$ as weights, properly accounting for the overlapping of intervals. We determine the frequency band contributing $99 \%$ of the sensitivity, as determined by the inverse variance. For the flat template $\Omega_{t}(f)=\Omega_{0}$, we estimate $\Omega_{0}=(-6.0 \pm 7.0) \times 10^{-4}$ in the frequency band $69-156 \mathrm{~Hz}$. The largest systematic errors are due to the calibration uncertainty of H1 (11\%) and of L1 (15\%). Figures 1 (right) and 2 (left) indicate that there are no dominating time-periods nor dominating spectral features contributing to this result. We construct a Bayesian posterior probability distribution for $\Omega_{0}$ based on the above measurement and on the uniform prior between 0 and 0.02 (corresponding to the maximum background that would still be consistent with 
the lowest interferometer strain noise), and we marginalize over the calibration uncertainties. This yields a Bayesian $90 \%$ upper limit on $\Omega_{0}$ of $\mathbf{8 . 4} \times \mathbf{1 0}^{-\mathbf{4}}$. Once the integrands in Eqs. 2 and 3 are known for the flat spectrum, it is a matter of simple scaling to generate the similar Bayesian $90 \%$ UL for any value of $\alpha$. The results are shown in Figure 2 (right).
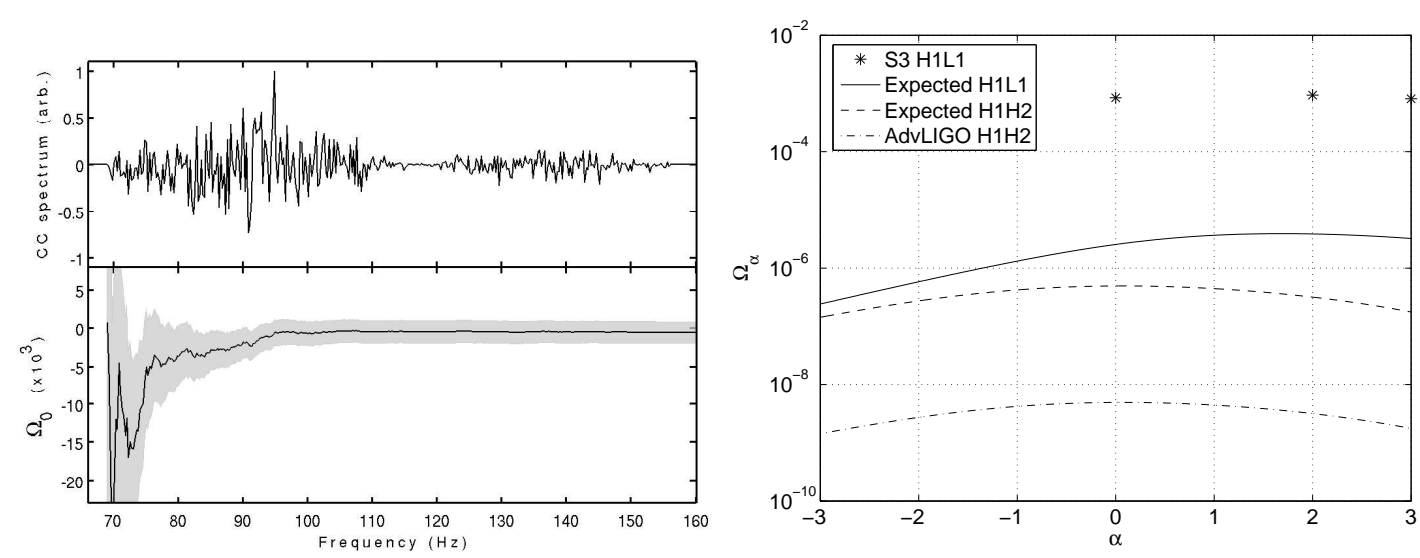

Figure 2: Left top: real part of the S3 H1-L1 cross-correlation spectrum, in arbitrary units. Left bottom: $\Omega_{0}$ estimate cumulative in frequency from $69 \mathrm{~Hz}$ to $f$. The shaded region shows the $\pm 2 \sigma_{\Omega_{0}}$ band. Right: Bayesian $90 \% \mathrm{UL}$ on $\Omega_{\alpha}$ as a function of $\alpha$ for the S3 result (for three values of $\alpha$ ), and expected sensitivities for the future LIGO runs (assuming design sensitivity and 1 year of exposure) and for Advanced LIGO (assuming 10 times better sensitivity than the Initial LIGO design, and 1 year of exposure). LIGO has recently started the science run S5, expected to reach the sensitivities of solid and dashed curves.

The authors gratefully acknowledge the support of the United States National Science Foundation for the construction and operation of the LIGO Laboratory and the Particle Physics and Astronomy Research Council of the United Kingdom, the Max-Planck-Society and the State of Niedersachsen/Germany for support of the construction and operation of the GEO600 detector. The authors also gratefully acknowledge the support of the research by these agencies and by the Australian Research Council, the Natural Sciences and Engineering Research Council of Canada, the Council of Scientific and Industrial Research of India, the Department of Science and Technology of India, the Spanish Ministerio de Educacion y Ciencia, the John Simon Guggenheim Foundation, the Leverhulme Trust, the David and Lucile Packard Foundation, the Research Corporation, and the Alfred P. Sloan Foundation.

\section{References}

[1] B. Abbot et al., Nucl. Instr. Methods A 517, 154 (2004).

[2] M. Maggiore, Phys. Rep. 331, 283 (2000).

[3] B. Allen, preprint (gr-qc/9604033).

[4] B. Allen and J. Romano, Phys. Rev. D 59, 102001 (1999).

[5] B. Abbot et al., Phys. Rev. D 69, 122004 (2004). 